\title{
A comunicação diaǵnóstica de ceratocone e sua influência na representação social que o paciente constrói da sua doença
}

\author{
The diagnostic communication of keratoconus and its influence on the social \\ representation that the patient has of his/her illness
}

\author{
Vera Lúcia Rodrigiues Alves ${ }^{1}$ \\ Milton Ruiz Alves ${ }^{2}$ \\ Silvia Tatiane Mauer Lane ${ }^{3}$
}

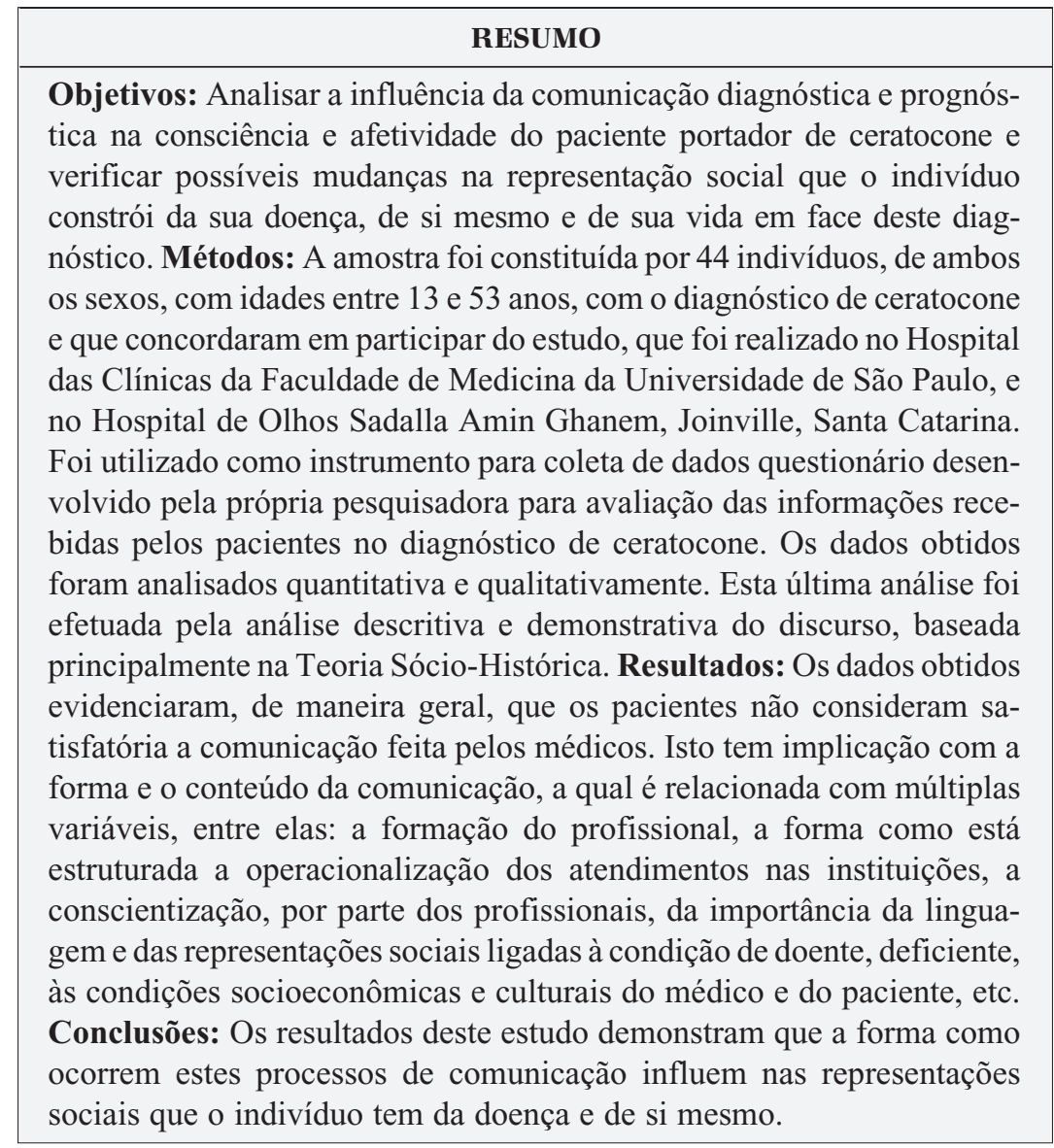

Descritores: Ceratocone/diagnóstico; Ceratocone/psicologia; Erros de refração/psicologia; Relações médico-paciente; Pacientes/psicologia; Comunicação

\section{INTRODUÇÃO}

A comunicação do diagnóstico e prognóstico das doenças interfere na dinâmica emocional dos pacientes de maneira geral. E como não é dado o devido cuidado a essa comunicação, gera distorções, mudanças e interferências na determinação social do próprio diagnóstico e na representação social que, o paciente tem de si mesmo, e do meio ao seu redor. Visto que a linguagem é constituinte e determinante da consciência, a comunicação 
pode interferir diretamente na consciência do indivíduo e nas suas emoções, determinando suas motivações e conseqüentes ações. Daí, a importância de se pesquisar como esse processo de comunicação acontece e como poderiam ser amenizados seus efeitos "negativos" na informação diagnóstica, dentro de um âmbito social maior ${ }^{(1)}$.

A aparente participação de diversos fatores no desenvolvimento do ceratocone contribui para as controvérsias, até hoje existentes, quanto a sua etiologia, hereditariedade, patogênese e bioquímica. Dos pacientes que apresentam ceratocone, 85 a $90 \%$ vão ter a afecção estabilizada entre 30 e 35 anos de idade. $\mathrm{Na} 1^{\underline{a}}$ fase o ceratocone induz à miopia e astigmatismo, sendo corrigido com óculos, em uma $2^{\underline{a}}$ fase com lentes de contato e evoluindo para $3^{\underline{a}}$ fase pode ser necessário transplante de córnea, ou seja, $5 \%$ a $15 \%$ poderão em algum momento necessitar de transplante de córnea para obter acuidade visual funcional ${ }^{(2)}$.

Cada vez mais tem se dado importância à comunicação diagnóstica das doenças, suas implicações sociais e qualidade de vida, entretanto, estudos especificamente para ceratocone são $\operatorname{poucos}^{(3-5)}$.

O ceratocone é causa de indicação de cerca de $50 \%$ dos transplantes de córnea realizadas no país e no mundo ${ }^{(6)}$. Rotineiramente quando o individuo tem este diagnóstico ele é estigmatizado como uma pessoa que deveria se matricular em "Banco de Olhos" para aguardar na fila a doação de uma córnea, o que ocorre muitas vezes pela desinformação médica sobre a evolução da doença. Esse futuro sombrio projetado sobre o paciente interfere no seu desenvolvimento e projeto de vida, em todas as instâncias quer seja física, psíquica, familiar e social.

Nesse aspecto o sentimento de medo como uma experiência social adquirida, mediante a comunicação, torna-se um inibidor e paralisador da consciência ${ }^{(7)}$. Visto que, em um primeiro momento, o medo aparece como uma forte emoção, mesmo que não esteja vinculado a uma ação imediata, e posteriormente, se canaliza em um afeto que passa a nortear as representações destes pacientes. $\mathrm{O}$ medo enquanto conhecimento prévio adquirido pela comunicação, transmitida por alguém que detém "poder", no caso o médico, tem uma força muito grande, porque este medo não é gerado por uma circunstância de risco iminente, em que a pessoa pode agir prontamente, mas ele é projetado no futuro. Melhor dizendo, é o medo gerado pela possibilidade de ficar deficiente podendo ou não ocorrer de acordo com as condições situacionais ao longo do tempo e são impossíveis cognitivamente de identificá-las no momento, portanto, desconhecido enquanto experiência concreta vivenciada, entretanto, com significados culturalmente conhecidos.

$\mathrm{O}$ afeto gerado por determinado conhecimento/informação dentro de um contexto médico-hospitalar pode levar a experiências determinantes e traumáticas para o indivíduo, levando a discussão e análise do paradigma médico no que se refere à postura, conhecimento e racionalidade médica. Esta possibilidade de abstração, de generalização, a partir da linguagem, da comunicação, ocorre na relação inter e intra-psíquica entre médico e paciente, em que a "consciência" é vista na relação do homem com a realidade e história social, vinculada ao trabalho, à linguagem e ao outro. Para Vygotsky ${ }^{(8)}$, a consciência está ligada ao desenvolvimento das palavras, as quais estão em constante processo de mudança, diferenciando o conceito de significado e sentido. Então se faz necessário refletir sobre as condições básicas de saúde, sobre os aspectos psicológicos, sociais e culturais e, na possibilidade de promoção de saúde e de transformação adaptativa da realidade, via comunicação diagnóstica e prognóstica da doença. Estas considerações ratificam a nossa percepção da necessidade de pesquisar subsídios para auxiliar essa relação e/ou pelo menos evidenciar na nossa sociedade estas dificuldades e sua relevância na saúde física, psíquica e social do paciente.

Esta pesquisa teve por objetivo analisar a influência da comunicação do diagnóstico e do prognóstico na consciência e afetividade do paciente portador de ceratocone; assim como verificar as possíveis mudanças na representação social que o indivíduo constrói de sua doença, de si mesmo e de sua vida em face deste diagnóstico.

\section{MÉTODOS}

Após a aprovação pela Comissão de Ética para Análise de Pesquisa - CAPPesq do Hospital das Clínicas da Faculdade de Medicina da Universidade de São Paulo realizou-se um estudo do tipo descritivo, exploratório, correlacional e explicativo com abordagem quantitativo-qualitativa. A amostra do estudo, do tipo aleatória, foi constituída por 44 indivíduos de ambos os sexos, com idades entre 13 e 53 anos, com diagnóstico de ceratocone, atendidos na seção de córnea e doenças externas no Hospital das Clínicas da FMUSP (São Paulo) e no setor de lentes de contato do Hospital de Olhos Sadalla Amin Ghanem (Joinville, SC) e que concordaram em participar do estudo. Foi adotado como instrumentos: entrevistas com pacientes e um questionário confeccionado para avaliação das informações recebidas pelos pacientes no diagnóstico de ceratocone, desenvolvido pela própria pesquisadora, contendo 12 questões para identificar variáveis relacionadas ao paciente (idade, sexo, escolaridade e profissão), ligadas à doença (motivo da procura do médico, doença que acreditava ter, como soube, idade que tinha e tempo de diagnóstico), ligadas ao diagnóstico (explicação incluiu menção à córnea, hereditariedade, cronicidade, indicação de lente, indicação de cirurgia); ligadas ao sentimento (sentimento, classificação do sentimento, influência hoje, influência no futuro e receio de limitação no futuro); ligadas à comunicação (procura de outro médico, informação que gostaria de receber e qualidade da comunicação recebida) (Quadro 1).

Os dados foram analisados quantitativa e qualitativamente. Esta última foi efetuada pela análise descritiva e interpretativa do discurso segundo a Teoria Sócio-Histórica associada a um estudo de caso. A análise quantitativa do tipo descri- 
tiva foi feita sobre as variáveis respostas e as variáveis do estudo. A análise quantitativa do tipo inferencial constituiu basicamente das análises de associação e de correlação. Para a realização adotaram-se os seguintes procedimentos estatísticos: intervalos de confiança de $95 \%$ para estimar porcentagens reais de alguns eventos de interesse do estudo; coeficiente de correlação linear de Pearson para avaliar a existência de dependência linear entre as variáveis de nível intervalar, e a correlação bisserial no caso de uma variável de nível intervalar e uma nominal dicotômica; e o teste de Qui-quadrado para comparação entre porcentagens de interesse em dois grupos independentes. Devido ao pequeno número de observações, as variáveis nominais foram dicotomizadas e sempre que observamos menos de $20 \%$ das freqüências esperadas inferiores a 5, a comparação foi feita por meio do teste exato de Fisher. Toda diferença, correlação ou associação com o valor de $\mathrm{p}$ inferior a 0,05 foi considerada estatisticamente significante.

\section{RESULTADOS}

A caracterização dos pacientes está na tabela 1 .

O exame da tabela 1 sugere para a variável sexo, predominância de pacientes do sexo feminino com $23(52,27 \%)$ citações, todavia, o teste de Qui-quadrado nos oferece evidência de que tal predominância não é estatisticamente significativa $(\mathrm{p}=0,878)$. Sugere para a variável idade, predomínio de pacientes com idade entre 19 e 24 anos com 14 (31,82\%) citações, valor médio de 25,98 (desvio-padrão de 8,81 ) e variação de 13 a 53 anos. A aplicação do teste de Qui-quadrado ofereceu evidência estatística da não homogeneidade da distribuição das idades $(\mathrm{p}<0,0001)$. Além disso, mais de $50 \%$ dos pacien-

\footnotetext{
Quadro 1 - Questões para avaliação das informações recebidas pelos pacientes portadores de ceratocone

Data:

Nome: .

Idade (data de nascimento):

Escolaridade (escola pública, particular):

Endereço (dos pais), telefone:

1- Por que você procurou esta Instituição?

2- Que doença você tem?

3- Como você ficou sabendo da doença?

4- Quantos anos você tinha quando soube da doença (diagnóstico)?

5- Qual diagnóstico o médico lhe deu?

6- O que o médico Ihe explicou a respeito do diagnóstico?

7- Como você se sentiu a respeito?

8- O que este diagnóstico/doença influi na sua vida hoje?

9- Em que você pensa que esta doença irá influir na sua vida futura?

10- Você receia vir a ter limitações ou impossibilidade de realizar alguma atividade no futuro?

11- Você procurou outros médicos, a respeito do seu diagnóstico e tratamento?

12- Que tipo de informação você gostaria de receber do médico?
}

tes tinham idade inferior a 24 anos. Sugere para a variável escolaridade, predominância do nível médio completo com $19(50,0 \%)$ e para a variável tipo de escola, predominância de escola pública com 29 (87,88\%) citações. A aplicação do teste de Qui-quadrado oferece evidência da não homogeneidade da distribuição da escolaridade $(p<0,001)$ e da distribuição da escola pública $(\mathrm{p}<0,001)$.

A distribuição das variáveis ligadas à doença está na tabela 2 .

Observou-se que os pacientes em sua maioria (41 pacientes) tinham conhecimento do nome da doença.

As classificações das explicações e respectivas composições estão na tabela 3 .

A tabela 3 sugere predominância de explicações insatisfatórias, com 37 (84,09\%) citações; de explicações que omitiram menção à alteração na córnea, com 26 (59,09\%) citações; de explicações que omitiram menção ao caráter hereditário da afecção, com 38 (86,36\%) citações; de explicações que omitiram menção ao caráter crônico da doença, com 36 (81,82\%) citações, de explicações que omitiram menção ao uso de lente de contato, com $29(65,91 \%)$ e que omitiram menção a recomendação de transplante, com 27 (61,36\%) citações. No campo inferencial estima-se, com $95 \%$ de confiança, que a real porcentagem média de explicações classificadas como satisfatória oscila de 4,66\% a 27,16\%; de explicações que incluem menção à alteração na córnea oscila de $25,79 \%$ a $56,03 \%$; de explicações que incluem menção ao caráter hereditário de $3,09 \%$ a 24,19\%; de explicações que incluem menção ao uso de lente de $19,51 \%$ a $48,67 \%$ e de explicações que incluem menção ao transplante de $23,67 \%$ a $53,61 \%$.

A caracterização dos sentimentos envolvidos está na tabela 4 .

\begin{tabular}{|c|c|c|}
\hline Variável & & Distribuição \\
\hline Sexo & $\mathrm{N}=44$ & $\%$ \\
\hline Masculino & 21 & 47,73 \\
\hline Feminino & 23 & 52,27 \\
\hline Idade & $\mathrm{N}=44$ & $\%$ \\
\hline 13 a 18 & 9 & 20,45 \\
\hline 19 a 24 & 14 & 31,82 \\
\hline 25 a 30 & 11 & 25,00 \\
\hline 31 a 36 & 2 & 4,55 \\
\hline 37 a 42 & 7 & 15,91 \\
\hline 43 a 48 & 0 & 0,00 \\
\hline 49 a 53 & 1 & 2,27 \\
\hline Escolaridade & $\mathrm{N}=38$ & $\%$ \\
\hline Fundamental incompleto & 8 & 21,05 \\
\hline Fundamental completo & 2 & 5,26 \\
\hline Médio incompleto & 2 & 5,26 \\
\hline Médio completo & 19 & 50,00 \\
\hline Superior incompleto & 1 & 2,63 \\
\hline Superior completo & 6 & 15,79 \\
\hline Tipo de escola & $\mathrm{N}=33$ & $\%$ \\
\hline Pública & 29 & 87,88 \\
\hline Não pública & 4 & 12,12 \\
\hline
\end{tabular}


No que se refere às influências, estima-se, com $95 \%$ de confiança, que a real porcentagem de pacientes que alegam que a doença influi hoje oscila de $63,59 \%$ a $89,89 \%$ e que alegam que a afecção irá influir no futuro oscila de $34,63 \%$ a $65,37 \%$. O exame da posição relativa entre as estimativas nos oferece evidência estatística de que a porcentagem de alegações de que a doença irá afetar o futuro é significativamente inferior ao das alegações de que a enfermidade afeta o presente.

A caracterização da comunicação está na tabela 5 .

No campo inferencial, estima-se, com $95 \%$ de confiança, que a real porcentagem de pacientes que procuram uma segunda opinião oscila de $54,47 \%$ a $83,63 \%$.

Na tabela 6 estão os valores dos coeficientes de correlação entre as variáveis quantitativas e qualitativas dicotômicas de interesse.

O exame dos dados contidos na tabela 6 nos oferece evidência estatística da presença de correlação positiva entre idade atual e idade que tinha; idade atual e tempo de diagnóstico; tempo de diagnóstico e sentimento; escore para a explicação e qualidade da explicação; escore para a explicação e informe da alteração da córnea; escore para a explicação e informe do caráter hereditário; escore para a explicação e informe da cronicidade; escore para a explicação e indicação de lente; escore para a explicação e indicação de transplante e escore para a explicação e qualidade da comunicação. Oferece evidência estatística da presença de correlação negativa entre idade e sexo; idade e motivo da procura; idade e indicação de transplante e tempo de diagnóstico e idade que tinha. Operacionalmente, os resultados sugerem que pacientes com

\begin{tabular}{|lcc|}
\hline \multicolumn{3}{|c|}{$\begin{array}{c}\text { Tabela 2. Distribuição das variáveis ligadas à doença. } \\
\text { (Questões 1 a 4) São Paulo, 2002. }\end{array}$} \\
Variável & $\mathrm{N}=44$ & Distribuição \\
Motivo da procura & 10 & 22,73 \\
Sintomas & 25 & 56,82 \\
Indicação & 7 & 15,91 \\
Campanha & 2 & 4,55 \\
Exame de rotina & $\mathrm{N}=44$ & $\%$ \\
Doenças que acredita ter & 41 & 93,18 \\
Ceratocone & 1 & 2,27 \\
Miopia, astigmatismo e ceratocone & 1 & 2,27 \\
Conjuntivite & 1 & 2,27 \\
Problema de visão & $\mathrm{N}=44$ & $\%$ \\
Como soube da doença & 26 & 59,09 \\
Rotina & 17 & 38,64 \\
Sintomas & 1 & 2,27 \\
Outros & $\mathrm{N}=44$ & $\%$ \\
Idade que tinha & 3 & 6,82 \\
3 a 10,9 & 14 & 31,82 \\
11 a 18,9 & 18 & 40,91 \\
19 a 26,9 & 4 & 9,09 \\
27 a 34,9 & 4 & 11,36 \\
35 a 41 & 5 & $\%$ \\
Tempo diagnóstico (anos) & $\mathrm{N}=43$ & 11,63 \\
Inferior a 7 & 34 & 6,98 \\
7 a 16,9 & 5 & 2,32 \\
17 a 20,9 & 3 & \\
Superior a 21 & 1 & \\
\hline
\end{tabular}

maiores tempos de diagnóstico tendem a apresentar sentimentos esperados; pacientes altos escores para a qualidade da explicação tendem a estar associados a pacientes que recebe-

\begin{tabular}{|c|c|c|}
\hline Variável & & Distribuição \\
\hline Classificação da explicação & $\mathrm{N}=44$ & $\%$ \\
\hline Insatisfatória & 37 & 84,09 \\
\hline Satisfatória & 7 & 15,91 \\
\hline IC 95\% para a \%Satisfatória & & $15,91 \pm 11,25$ \\
\hline $\begin{array}{l}\text { Explicação inclui menção à } \\
\text { alteração na córnea }\end{array}$ & $\mathrm{N}=44$ & $\%$ \\
\hline Não & 26 & 59,09 \\
\hline Sim & 18 & 40,91 \\
\hline IC $95 \%$ para a \%Sim & & $40,91 \pm 15,12$ \\
\hline $\begin{array}{l}\text { Explicação incluiu menção } \\
\text { à hereditariedade }\end{array}$ & $\mathrm{N}=44$ & $\%$ \\
\hline Não & 38 & 86,36 \\
\hline Sim & 6 & 13,64 \\
\hline IC 95\% para a \%Sim & & $13,64 \pm 10,55$ \\
\hline $\begin{array}{l}\text { Explicação incluiu menção } \\
\text { à cronicidade }\end{array}$ & $\mathrm{N}=44$ & $\%$ \\
\hline Não & 36 & 81,82 \\
\hline Sim & 8 & 18,18 \\
\hline IC 95\% para a \%Sim & & $18,18 \pm 11,86$ \\
\hline $\begin{array}{l}\text { Explicação incluiu indicação } \\
\text { de lente de contato }\end{array}$ & $\mathrm{N}=44$ & $\%$ \\
\hline Não & 29 & 65,91 \\
\hline Sim & 15 & 34,09 \\
\hline IC 95\% para a \%Sim & & $34,09 \pm 14,58$ \\
\hline $\begin{array}{l}\text { Explicação incluiu indicação de } \\
\text { transplante }\end{array}$ & $\mathrm{N}=44$ & $\%$ \\
\hline Sim & 27 & 61,36 \\
\hline Não & 17 & 38,64 \\
\hline IC 95\% para a \%Sim & & $38,64 \pm 14,97$ \\
\hline $\mathrm{IC}=$ intervalos de confiança & & \\
\hline
\end{tabular}

\begin{tabular}{|lcc|}
\hline \multicolumn{3}{|c|}{$\begin{array}{c}\text { Tabela 4. Distribuição dos sentimentos envolvidos. } \\
\text { (Questões 7 a 10). São Paulo, 2002. }\end{array}$} \\
Variável & $\mathrm{N}=44$ & Distribuição \\
Sentimento & 3 & $\%$ \\
Contraditório & 36 & 6,82 \\
Depressivo & 5 & 81,82 \\
Indiferente & $\mathrm{N}=43$ & 11,36 \\
Influência hoje & 10 & $\%$ \\
Não & 33 & 23,26 \\
Sim & & 76,74 \\
IC 95\% para a \%Sim & $\mathrm{N}=36$ & $76,74 \pm 13,15$ \\
Quanto influencia hoje & 15 & $\%$ \\
Muito & 11 & 41,67 \\
Pouco & 10 & 30,56 \\
Médio & $\mathrm{N}=36$ & 27,78 \\
Influência no futuro & 22 & $\%$ \\
Não & 22 & 50,00 \\
Sim & & 50,00 \\
IC 95\% para a \%Sim & $\mathrm{N}=44$ & $\%, 00 \pm 15,37$ \\
Receia limitações no futuro & 18 & 40,91 \\
Não & 2 & 4,55 \\
Não sabe & 24 & 54,55 \\
Sim & & \\
IC= intervalos de confiança & & \\
\hline
\end{tabular}


ram informação quanto à alteração na córnea, hereditariedade, cronicidade da afecção e indicação de lente e transplante. Observa-se também que pacientes mais idosos tendem a ter procurado o médico por apresentar sintomas e ter recebido indicação de transplante.

\section{DISCUSS ÃO}

Os dados apresentados nesta análise, junto aos 44 pacientes de ceratocone, demonstram que sob a ótica deles, as

\begin{tabular}{|lcc|}
\hline \multicolumn{2}{|c|}{$\begin{array}{c}\text { Tabela 5. Distribuição das variáveis ligadas à comunicação. } \\
\text { (Questôes 11 e 12) São Paulo, 2002. }\end{array}$} \\
Variáveis & $\mathrm{N}$ & Distribuição \\
Procurou outros médicos & 13 & $\%$ \\
Não & 29 & 30,95 \\
Sim & & 69,05 \\
IC 95\% para a \%Sim & 44 & $69,05 \pm 14,58$ \\
Tipo de informação & 6 & $\%$ \\
Outra & 10 & 13,64 \\
Doença & 6 & 22,73 \\
Tratamento & 17 & 13,64 \\
Nenhuma & 5 & 38,64 \\
Doença e tratamento & 44 & 11,36 \\
Qualidade da comunicação & 8 & $\%$ \\
Outras & 19 & 18,18 \\
Insatisfatória & 17 & 43,18 \\
Satisfatória & & 38,64 \\
\hline
\end{tabular}

informações recebidas dos profissional-médicos são consideradas insatisfatórias e não atendem suas necessidades globais.

Neste estudo não houve prevalência de gênero, mas sim predomínio do sexo feminino e de adolescentes e adultos jovens, a maioria provenientes de escolas públicas, todos com algum grau de escolaridade, predominando o nível médio (Tabela 1). Observou-se que os pacientes em sua maioria (41 pacientes) tinham conhecimento do nome da afeç̧ão (Tabela 2). No entanto, a explicação e conseqüente conhecimento, acerca do que é a doença, dos aspectos clínicos e tratamento foram considerados insatisfatórios por 37 pacientes (Tabela 3). A vivência de sentimentos, caracterizados como contraditórios, depressivos e indiferentes, em face de um diagnóstico desta natureza foi referida por 36 pacientes (Tabela 4).

Estes resultados apontam para as representações sociais coletivas da condição de doente, de deficiente na nossa cultura e da importância de se ter um cuidado maior, quando se está no papel de emissor/transmissor de informações tão carregadas de conteúdo emocional e ideológico que é a vivência da condição de doente e da confrontação com os preconceitos ligados a esta condição em termos pessoais e sociais. Corroborando para a necessidade de maior instrução a ser fornecida ao paciente, sabemos que o medo relativo ao risco pode ficar sensivelmente ampliado pelo desconhecimento dos limites de risco ou pela ignorância dos métodos de prevenção eficazes ${ }^{(9)}$, e minimizados em face de uma melhor adequação ao tratamento ${ }^{(1,10)}$.

O fato da comunicação diagnóstica não ter sido vista como satisfatória para um número expressivo de pacientes (Tabela 5)

\begin{tabular}{|c|c|c|c|}
\hline & Idade & Tempo diagnóstico & Escore para explicação \\
\hline Sexo masculino & $-0,51^{*}$ & $-0,09$ & $-0,12$ \\
\hline Idade & 1,00 & $0,39^{*}$ & $-0,20$ \\
\hline Escola fundamental incompleta & 0,10 & 0,12 & $-0,03$ \\
\hline Escola pública & 0,02 & 0,04 & 0,00 \\
\hline Procura por sintoma & $-0,29^{*}$ & $-0,06$ & 0,12 \\
\hline Doença ceratocone & 0,17 & $-0,10$ & 0,03 \\
\hline Soube por exame de rotina & 0,12 & $-0,15$ & 0,22 \\
\hline Idade que tinha & $0,71^{*}$ & $-0,36^{*}$ & $-0,07$ \\
\hline Tempo de diagnóstico & $0,39^{*}$ & 1,00 & $-0,18$ \\
\hline Diagnóstico inicial de ceratocone & 0,16 & $-0,16$ & 0,13 \\
\hline Escore para a explicação & $-0,20$ & $-0,18$ & 1,00 \\
\hline Explicação satisfatória & $-0,13$ & $-0,14$ & $0,72^{*}$ \\
\hline Informou alteração da córnea & 0,12 & 0,07 & $0,41^{*}$ \\
\hline Informou hereditariedade & $-0,11$ & $-0,24$ & $0,31^{*}$ \\
\hline Informou cronicidade & $-0,23$ & $-0,23$ & $0,50^{*}$ \\
\hline Indicou lente & $-0,06$ & $-0,12$ & $0,66^{*}$ \\
\hline Indicou transplante & $-0,25^{\star}$ & $-0,01$ & $0,56^{*}$ \\
\hline Sentimento esperado & 0,15 & $0,39^{*}$ & $-0,03$ \\
\hline Influência hoje & 0,02 & $-0,17$ & 0,07 \\
\hline Influência no futuro & $-0,15$ & $-0,19$ & 0,00 \\
\hline Procurou outro médico & $-0,20$ & 0,05 & 0,03 \\
\hline Quer saber mais sobre tratamento & 0,03 & 0,17 & $-0,24$ \\
\hline Comunicação satisfatória & 0,09 & 0,00 & $0,30^{*}$ \\
\hline
\end{tabular}


pode estar relacionada a fatores como não conhecimento por parte do médico, a quem se dá o diagnóstico; a forma e o conteúdo da linguagem utilizada; a formação profissional e a identificação com os pressupostos éticos da relação médicopaciente e instituição. O nível de informação que os pacientes avaliados referem ter aponta, salvo algumas exceções, para um comportamento "técnico e/ou especializado" por parte dos médicos, pois os pacientes conhecem o nome de sua doença e sua gravidade em última instância, como por exemplo: não poder dirigir, estudar, etc, e na possibilidade de transplante de córnea como forma de evitar a cegueira, embora o ficar cego, não seja dito claramente, mas sim inferido pela maioria dos pacientes. Porém, o desconhecimento da doença em si, quanto a sua etiologia, hereditariedade, aspectos clínicos, formas de tratamento e a necessidade e o desejo de ter maiores informações, assim como a referência de "repercussão negativa" em suas vidas, apontam para a precariedade desses informes e explicações (Tabela 6).

Para Vygotsky (1934)*, citado por van der Veer e Valsiner ${ }^{(11)}$, "o pensamento não é apenas externamente mediado por signos, mas também internamente por significados. A questão é que a comunicação direta de mentes é impossível, não só fisicamente, mas também psicologicamente. Ela só pode ser conseguida por meios indiretos, mediados. Esta estrada equivale à mediação interna do pensamento primário por significados, depois por palavras. Portanto, o pensamento nunca pode ser igual ao significado direto das palavras. $\mathrm{O}$ significado media o pensamento em seu caminho para a expressão verbal, ou seja, o caminho do pensamento para a palavra é um caminho indireto, inteiramente mediado". Destaca-se a necessidade do médico se auto-perceber e ter consciência crítica de sua atuação profissional, bem como de se preparar para receber o outro, foco e receptor de sua atuação clínica e conhecimento. Pois a não percepção ou falta de cuidado por parte dos profissionais da importância e significado desta comunicação, quer na forma de linguagem utilizada e da influência que esta pode gerar no outro (paciente), nos aspectos cognitivos, afetivos emocionais e na própria consciência de si, assim como nos seus relacionamentos sociais, será decisivo para se estabelecer uma boa comunicação e/ou encontro respeitoso e ético.

Através do próprio discurso constante nas respostas relativas às questões $6 \mathrm{a} 12$ (Quadro 1), verificamos que a influência do diagnóstico pode ocasionar limitações de práxis funcionais, cotidianas (atividades de auto-cuidado, de trabalho e de lazer) e nas relações com o outro, que estão diretamente ligadas aos sentimentos e emoções decorrentes da comunicação da doença. Podemos constatar que a projeção de limitações no futuro (22 pacientes) influi na conscientização que estes sujeitos tem da doença, do tratamento, do profissional que os atende e da instituição hospitalar.

\footnotetext{
"Vygotsky (1934) apud van der Veer R, Valsiner L. ${ }^{(11)}$
}

Pensar a relação médico-paciente como um diálogo que permite entre outras coisas a conscientização e a melhora de qualidade de vida é quase inerente nesta interação. Entretanto, podemos verificar que dependendo de como ela se estabelece, pode gerar efeitos adversos. O que nos impele a buscar na formação médica, enquanto especialista e formadores de opiniões, a rever conceitos, visando no desempenhar seu papel profissional, ampliar seus objetivos em relação ao outro/paciente e colaborar para o processo de desenvolvimento bio-psico-social, pela relevância de sua atuação junto à sociedade. Evidenciando-se que mesmo em face da deficiência, é possível colocá-la de tal maneira que estimule a necessidade de compensação de forma integrada e produtiva. Esta responsabilidade recai sobre o profissional, face à sua função de diagnosticar, orientar e coordenar o tratamento com vistas à superação e enfrentamento das dificuldades dentro de uma inserção social maior.

\section{CONCLUSÃO}

Neste estudo verifica-se a preocupação com a inserção social, relacionada com o diagnóstico de ceratocone e 76\% dos pacientes sentem que ele influiu em sua vida diária (no auto-cuidado, escola, trabalho e lazer), porém, com percepções diferentes de quanto isto afeta a vida como um todo. $\mathrm{O}$ que nos parece ser um denominador comum é a comunicação, que amplia as possibilidades de enfrentamento deste diagnóstico, assim como pode influenciar na representação social de maneira mais assertiva e menos iatrogênica, propiciando uma melhor qualidade de vida aos seus portadores e estimulando sua autonomia, auto-estima e, sua inclusão social.

\section{ABSTRACT}

Purpose: To analyze the influence of diagnostic and prognostic communication and the awareness and affectivity of the patient with keratoconus and to verify the possible modifications in the social representation that the individual has of the disease, of himself and of his life. Methods: The sample was consisted of 44 subjects, both genders, aged 13 to 53 years, with diagnosis of keratoconus who agreed to participate in this study. The study was carried out at the "Hospital das Clínicas", University of São Paulo Medical School, and at the "Hospital de Olhos Sadalla Amin Ghanem" (Eye Hospital), in Joinville, state of Santa Catarina. A questionnaire used to assess the information received by patients with keratoconus was specially developed for this study. A quantitative and qualitative analysis of the collected data was performed through descriptive and demonstrative analyses of speech, based mainly on Social-Historical Theory. Results: In general, the patients did not consider the information provided by the doctors as being satisfactory. This fact may be related to how the information is transmitted and its contents. This is influenced by several 
variables, such as professional training, structural characteristics of the provided care, awareness by the doctor of the importance of language and social representation related to the condition of the patient, the socioeconomic and cultural level of the physician and the patient, etc. Conclusion: This study indicates that the way the information is transmitted influences the social representation that the individual has of the illness and of himself.

Keywords: Keratoconus/diagnosis; Keratoconus/psychology; Refractive errors/psychology; Physician-patient relations; $\mathrm{Pa}$ tients/psychology; Comunication

\section{REFERÊNCIAS}

1. Alves VLR. O desconhecimento pelos pais do diagnóstico de deficiência física em crianças portadoras de retardo no desenvolvimento neuropsicomo- tor, sua influência no programa de reabilitação [tese]. São Paulo: Pontifícia Universidade Católica de São Paulo; 1997.

2. Bechara SJ, José NK. Ceratocone. In: Belfort Júnior R, José NK. Córnea: clínica crúrgica. São Paulo: Roca; 1997. p.359-66.

3. Lima CA. Proposição e teste de um questionário de qualidade de vida em pacientes com ceratocone [tese]. Campinas: Faculdade de Ciências Medicas da Universidade Estadual de Campinas; 2000.

4. Alves VLR. A comunicação diagnóstica de ceratocone e a sua influência na representação social que o paciente constrói da sua doença [tese]. São Paulo: Pontifícia Universidade Católica de São Paulo; 2003.

5. Moreira LB. Aspectos psicossociais do paciente com ceratocone [tese]. São Paulo: Universidade Federal de São Paulo. Escola Paulista de Medicina; 2006.

6. Milani JAA. Transplante de córnea: Conceitos básicos. In: Lima ALH, Nishiaki-Dantas MC, Alves MR. Doenças externas oculares e córnea. Rio de Janeiro: Cultura Médica; 1999. p.435-57.

7. Heller A. Teoria de los sentimientos. Barcelona: Fontamara; 1985.

8. Vygotsky LS. Pensamento e linguagem. São Paulo: Martins Fontes; 1993.

9. Dejours C. A loucura do trabalho: estudo de psicopatologia do trabalho. $4^{\mathrm{a}}$ ed. São Paulo: Cortez; 1995. p.66.

10. Cavalcanti FS, Freitas GG, Araújo MHQ, Leite MSS, Castro BTD. Nível de informação fornecida ao paciente sobre sua doença. Rev Bras Reumatol. $1993 ; 33(6): 223-5$.

11. Van der Veer R, Valsiner J. Vygotsky: uma síntese. São Paulo: Loyola; 1996. p.134. 